\title{
A Resolution Anlaysis of Two Geophysical Imaging Methods for Characterizing and Monitoring Hydrologic Conditions in the Vadose Zone
}

\author{
David L. Alumbaugh \\ Department of Civil and Env. Eng. \\ University of Wisconsin-Madison \\ 2258 Engineering Hall \\ 1415 Engineering Drive \\ Madison, WI 53706
}

\section{Project Objectives:}

This project has been designed to analyze the resolution of two different geophysical imaging techniques (electrical resistivity tomography and cross-borehole ground penetrating radar) for monitoring subsurface flow and transport processes within the vadose zone. This is to be accomplished through a coupled approach involving large scale unsaturated flow modeling, petrophysical conversion of the resulting 2 and 3 Dimensional water content and solute concentration fields to geophysical property models and generation of synthetic geophysical data, followed by the inversion of the synthetic geophysical data. The resolution, strengths, and limitations of the geophysical techniques will then be ascertained through an analysis involving comparisons between the original hydrologic modeling results and inverted geophysical images. Increasing levels of complexity will be added to the models as the project progresses through the addition of heterogeneity in the original hydrologic property model, and by adding uncertainty to the petrophysical relationship that couples the geophysical model to the hydrologic modeling results.

\section{Progress:}

To date much of the focus has been on the unsaturated flow modeling, including defining the hydrologic properties and their distributions within the models. Most of the work to date has involved running 2D and 3D simulations with straightforward homogenous and layered property distributions. We are beginning to work on developing more complex hydrogeologic property models, particularly for the 3-D modeling which requires using parallel computing resources at Sandia National Labs. A simple six-layer model has been designed to crudely simulate subsurface conditions at the Sandia-Tech Vadose Zone facility where two previous EMSP funded experiments were completed (EMSP Projects 55332 and 70267; Brainard et al., 2003, 2004). The hydrologic properties that have and are being incorporated into the model have used both laboratory measurements (Baker, 2002) as well as a pedo-transfer function approach (Chang, 2003). Because the latter correlates grain-size distribution to hydraulic properties, we have recently begun a detailed grain-size analysis of continuous core collected at the site using a laser device that is available at the Sandia National Laboratory facilities in Carlsbad, New Mexico. This will also provide us additional information in the spatial variability at the site, at least in the vertical direction. In hydrologic modeling activities we have focused on developing basic one-, two-, and three-dimensional (1D, 2D, and 3D) models. The 1D and 2D simulations have been conducted with HYDRUS-2D (Simunek J., and van Genuchten ,1999) and iTOUGH2(Finsterely, 1997), and have allowed for efficient examination of optimal grid resolution as well as examination of initial and boundary condition effects on simulated water content fields. These simulations have also provided a foundation for development of more complex 3-D heterogeneous hydraulic property fields by allowing us to 1) constrain the range of hydraulic property parameters, 2) place limits on contrasting hydraulic properties between adjacent layers, and 3) to identify initial conditions limits in the dry end of the range given a variety of near surface hydrologic property parameters and boundary conditions. These constraints were chosen based on the numerical convergence efficiency. Several 3-D PorSalsa (Shadid et al., 1996) simulations, incorporating single-layer homogeneous, six-layer homogenous, and randomly heterogeneous hydraulic property fields, grid spacing on the order of $0.25 \mathrm{~m}(137,677$ nodes), have recently been completed. We are currently compiling the simulations? results to be transferred to other members of the research team. These simulations were performed on a relatively small 32-node parallel Linux cluster. The limited memory on this cluster does not 
allow us to examine finer resolution cases. Subsequently, we have compiled PorSalsa on a much larger parallel cluster, which we plan to use for problems with grid spacing on the order of $0.1 \mathrm{~m}$. We are currently in the process of testing PorSalsa on the bigger cluster. Finally, we are also investigating various methods to incorporate spatially correlated property fields, generated using commercial packages such as GSLIB, into PorSalsa as well as iTOUGH2 codes. Two-dimensional electromagnetic property fields were assembled using results from Hydrus-2D along with a simple petrophysical relationship that approximates conditions at the Soccoro site. Note that the petrophysical relationship was considered to be exact rather than being stochastic in nature. This model has in turn been employed to compute synthetic cross-borehole GPR data using the scheme given in Holliger and Bergman (2002). Initial results indicate that velocity/moisture content images resulting from straight ray inversion of the data using the Geotom algorithm (Jackson and Tweeton, 1996; Geotom, 1998) recover the true model fairly well, although curved ray inversion does somewhat better. However, straight ray inversion of the GPR amplitudes to produce images of attenuation is not as robust. A simple comparison to images constructed from synthetic data computed with a straight ray inversion algorithm suggest that the poor reconstruction is primarily due to the straight ray far-field assumptions within the inversion algorithm not properly accounting for near-field curved ray propagation. In any event, the simulations have shown that care must be taken when interpreting the attenuation and velocity images together to avoid over-interpreting artifacts as true structure. While we wait for more detailed 3D modeling results, the Hydrus-2D results are also being used for initial 3D modeling of ERT data. Models are constructed by assuming the hydrological parameters are constant in the direction perpendicular to strike. A local, empirical model was developed for the pre-infiltration Socorro site by comparison of neutron derived moisture contents with borehole induction logs. This model is used to convert the Hydrus2D models to synthetic resistivity sections with a very fine cell size, approximately $6.5 \times 6.5 \times 6.5 \mathrm{~cm}$. The resulting forward models require very large meshes, several million elements, to approximate field scale surveys. These forward models are then inverted using the same methods and parameters applied to field data. The initial results have shown that care must be taken in the design of the meshes used for the creation of these very large forward models to assure accuracy of the resulting models.

\section{References:}

Baker, K. E., 2002, Investigation of direct and indirect hydraulic property laboratory characterization methods for heterogeneous alluvial deposits: Application to the Sandia-Tech vadose zone infiltration site, Master thesis, Department of Earth and Environmental Science, New Mexico Institute of Mining and Technology, Socorro, NM, 137 pp.

Brainard, J. R., D. L. Alumbaugh, D. J. La Brecque, T.C. J. Yeh, P.Y. Chang, and L. M. Hall. 2004. The Sandia-Tech Vadose Zone Facility, experimental design and data report of tracer experiments. Sandia Internal Report In Progress. Sandia National Laboratories, Albuquerque, NM.

Brainard, J. R., R. J. Glass, D. L. Alumbaugh, L. Paprocki, D. J. La Brecque, X. Yang, T.-C. J. Yeh, K. E. Baker and C. A. Rautman, 2004, The Sandia-Tech Vadose Zone Facility; Experimental Design and Data Report of a Constant Flux Infiltration Experiment; Sandia National Laboratories Report.

Chang, P.Y., 2003, An analysis of a coupled geophysical tomography -hydrological modeling method to characterize hydraulic properties, and flow and transport process in the vadose zone; $\mathrm{PhD}$ Thesis, Geological Engineering Program, University of Wisconsin-Madison. GeoTom, LLC.,1998, User Mannual for GeotomCG and GeoTom3D, GeoTom, LLC., Apple valley, Minnesota.

Holliger, K., and Bergmann, T., 2002, Numerical modeling of borehole georadar data, Geophysics, Vol 67, No. 4, p. 1249-1257. Finsterle, F., 1997. ITOUGH Command Reference, Earth Sciences Division, Lawrence Berkeley National Laboratory, University of California, Berkeley, CA 94720.

Jackson, M., J., Tweeton, D., R., 1996, 3DTOM: Three-dimentional geophysical tomography, Report of investigations 9617, Bureau of Mines, United Stated Department of the Interior., pp. 1-84. 
Shadid, J. N., A. G. Salinger, R. C. Schmidt, T. M. Smith, S. A. Hutchinson, G. L. Hennigan, K.D. Devine, and H. K. Moffat, MPSalsa Version 1.5: A Finite Element Computer Program for Reacting Flow Problems, SAND98-2864, Sandia National Laboratories, Albuquerque, New Mexico, 1999.

Simunek J., and van Genuchten, M. Th., 1999, The HYDRUS-2D software package for simulating the Two-Dimensional Movement of water, heat and multiple solutes in variably-saturated media, Version 2.0, U. S. Salinity Laboratory, U.S. Department of Agriculture, Riverside, CA. 227p. 